\section{Briginal \$lluticleg.}

THE ADVISABILITY OF INDUCING ABORTION IN CASES OF SO-CALLED UNCONTROLLABLE VOMITING OF PREGNANCY.1

BY o. W. DOE, M.D.

The rarity of this disease naturally prevents one from deducing principles on which to base his method of practice from his own experience. This is especially so with me, as I have seen only one indisputable case during the past twelve years.

Consequently in this paper my deductions are drawn from a compilation of all the undoubted cases that could be found referred to in the Index Medicus and various works on obstetrics.

In looking up the various references I want to publicly acknowledge my great indebtedness to Dr. S. H. Ayer, for the very valuable assistance he has given me.

At a recent meeting of the Boston Obstetrical Society I reported a case of so-called uncontrollable vomiting of pregnancy, which was relieved by changing the malposition of the uterus after almost every remedial agent had been unsuccessfully tried and the patient's condition had become most alarming.

In consultation, the question of inducing abortion arose and it was certainly a most difficult and anxious one to decide. The patient had taken no nourishment by the mouth for nearly five weeks, as even the mention of food would excite vomiting. During this time she had been fed by nutritive enemata. Nausea was constant and intense, and vomiting of a thin yellowish fluid, sometimes half a cupful at a time, occurred every two or three hours. Any sudden noise, or sudden appearance of one by the bedside would excite vomiting.

Notwithstanding the fact that the enemata had been well born, the patient had become greatly emaciated and too weak to move herself in bed.

'There was excessive ptyalism, coming on more frequently at night; at other times thirst was intense, tongue parched and dry, breath very offensive, and, along the course of the cesophagus and at the epigastrium, was marked tenderness. Pulse 116, temperature $97^{\circ}$.

The cervix was firm and unyielding; the os only slightly patulous. 'The uterus was markedly anteverted, the fundus resting against the rami of the pubes, and, on examination, could be raised up out of the cavity of the pelvis, but fell back again directly upon the removal of the finger. She was within three or four days, as near as could be determined, of being three months advanced. Taking into consideration the unyielding and firm condition of the cervix, I reasoned that were abortion to be induced she was far too weak to bear the shock necessarily attending it, and even if she happily withstood this there was the probability of hæmorrhage, which, in one so weak, would at its very outset prove fatal. Furthermore, should she surmount even these two dangers, there still remained a third, namely, septicæmia, which one would almost expect to follow in a person so reduced when abortion was artificially and forcibly produced. In favor of delay and trusting to other means was the fact that in her preceding pregnancy the nausea and vomiting though much less severe, yielded suddenly and completely: at the bof of the fourth month after the attending $p$ t pil in had on vaginal examination, by chatee raisedfle haterus high up out of the cavity of the pelyis. Futhermare there was no continued irritability of thowely that, as a rule, the enemata were well $\mathrm{Had}$ at this time from any cause rectal alimentation been impracticable, and the elevation of the uterus and retention by vaginal support proved inefficient, even in the face of every threatening danger, I should have advised rapid abortion under ether and full hypodermic stimulation. Should the result have proved fatal, I should have considered that the error in treatment ought to be referred to the delay, rather than to the method employed.

The exciting cause of the vomiting in this case seemed to be wholly the displacement of the uterus and consequent pressure upon the pelvic parts. As soon as the pressure was permanently removed, by raising the body of the uterus out of the pelvic cavity, the vomiting and nausea instantly ceased. A peculiar feature illustrating the principle of cause and effect here was that as soon as the vaginal tampons were at all displaced, so as to allow the body to prolapse or antevert, the nausea and vomiting would directly return. Looking back upon this case and noting each stage of the disease as it unfolded itself, and remembering how inefficient all therapeutic agents proved, I should be inclined to follow in another case a course of treatment like the following : rectal alimentation combined with chloral and bromide as soon as the vomiting became pronounced; careful examination to detect any malposition of the uterus and as soon as observed to remedy it by the use of a proper pessary and with the tamponing of the vagina with the object of raising the body as far out of the pelvis as possible. Should no relief follow soon after the beginning of the fourth month, or if even earlier no relief followed the correction of the displacement, and the patient's strength was rapidly diminishing, or if rectal irritation became continuous, I should unhesitatingly induce abortion. Or, more concisely, impracticable rectal alimentation combined with continuous vomiting, wholly unrelieved by correcting any existing uterine displacement, attended with rapid pulse, offensive breath, subnormal temperature and marked increasing prostration would hereafter decide me to induce abortion at whatever the period of pregnancy might be.

Under the conditions I have just expressed there is no doubt in my mind as to the advisability of inducing abortion in cases of so-called uncontrollable vomiting of pregnancy, still it will be but fair to present to you the various differences of opinion upon the subject. Every physician has probably either seen or read of cases of death from this terrible complication of pregnancy, and yet many eminent practitioners say that abortion without exception is entirely unnecessary. Dr. Mortimer H. Jordan, of Birmingham, Ala, says : ${ }^{2}$ "Among other eminent men I will mention Cazeaux, Burns, Dubois, Desermaux, Carl Braun, of Vienna, and Henry F. Campbell, of Augusta, Ga., who state that artificial production of abortion is never justifiable for gravid nausea." Dr. Campbell says : "That under the careful and systematic application of rectal alimentation artificial abortion for the relief of gravid nausea can be banished from practice even as a last resort." On the other hand, I quote from Dr. Davenport as follows: 8 "Denman, Merriman, Burns, 
Davis, Lee, Ashwell, Blundell, Churchill and Condie eaux and others have arrived is that under no circumall advocate the induction of premature labor, without stances are we justified in inducing premature labor reference to the period of gestation; if the symptoms for the relief of vomiting of pregnancy." Cazeaux and condition of the patient demand it, then it is to reports seven cases of induced abortion with six be done." J. Marion Sims writes as follows :" "Dur- deaths. According to his estimate, one in seven is ing my residence in Paris some years ago, I saw two the average of escapes from death after the operation deaths from pregnancy-vomiting, and I saw two cases (induced abortion). He thinks the most desperate that were snatched from the jaws of death by the in- cases, as he cites several to show, have a better chance duction of abortion. Professor. Trousseau and Dr. on the expectant plan, with such nutriments as can Johnston of Paris were consulted in these cases and be retained, than in the resort to induced abortion. both agreed that prompt abortion was the only means Out of 118 cases collected by Gueniot there were of saving life." Dr. Murphy sums up four cases as 72 recoveries and 46 deaths, represented as follows:

follows: " Thus in four cases of excessive vomiting followed by exhaustion, three were lost through hesitation and delay; and one was saved by promptitude in inducing miscarriage."

Lusk says: ${ }^{6}$ "When the vomiting is literally uncontrollable, a rare event where the physician commands the full coöperation of his patient, and death from starvation threatens, there remains as an ultimate resource the artificial induction of abortion or premature labor.

Dr. Scott says : ${ }^{7}$ "It is criminal to allow a patient to go to the last extremity before an effort is made to produce an effect which we know is certain to relieve her and preserve a life which is the 'right of woman.' " On the other hand, however, cases are recorded where the patient sank steadily and in which life had been despaired of by the physician in charge, and in which abortion had been refused by the patient, and yet upon the exhibition of some new remedy, a change for the better has appeared and a steady gain made from that time on. P. Dubois ${ }^{8}$ speaks of a case which illustrates this point, it being one which had been abandoned by himself and colleague and which afterward promptly recovered on the exhibition of a purgative.

Dr. Engelmann says : ${ }^{\ominus}$ "Of course abortion is to be induced as a last remedy, but it is always to be used in time, and as soon as it is found that all others fail." The principal fact is, practitioners wait too long. Dr. Coles says : ${ }^{10}$ "I have never had a case in my own practice where the induction of labor was necessary, but I have seen two cases where this had to be resorted to. In these cases the women were in extremis; one of them died notwithstanding the abortion. She had gone too far. We had waited toolong for this spontaneous change."

Leishman says: " 11 The conclusion at which Caz-

Without abortion in very severe cases after diversifled treatment. severe cases
ter abortion or induced labor in cases fter abortion or induced labor in cases
more or less desperate . . . Total, Recoveries. Deaths. $31 \quad 28$ 207

$\frac{21}{72} \quad \frac{11}{46}$

Dr. Alfred McClintock in a paper before the Dublin Obstetrical Society read in 1873, reported 36 cases in which abortion was induced with 27 recoveries and nine deaths.

In selecting the following 48 cases, which I am about to report, there was considerable difficulty experienced in deciding whether a case should be truly called "uncontrollable" in the popular sense of the word, or simply obstinate. It is evident, however, that all cases in which death occurred without artificial abortion were certainly uncontrollable, and that by adding to the list of recoveries, cases which were more or less doubtful, the percentage of deaths became correspondingly less.

All of the cases are very briefly reported, some from necessity, others from choice, the idea being not to bring forward a full and complete report of each case, but simply to place before you such points as have a direct bearing on the subject of this paper. I think, however, the principal point to be brought out is clearly shown; namely, that after the emptying of the uterus, whether artificially or otherwise, the vomiting, with very few exceptions, soon ceased, and that in nearly all cases recovery took place, a fatal termination occurring in those in which interference had been too long deferred.

I shall first present to you twenty-eight cases of uncontrollable vomiting, in which artificial abortion was not induced, after which I shall read twenty cases which were terminated by artificial abortion.

Twenty-Eight Cases of Uncontrollable Vomiting of Pregnancy, in which Artificial Abortion WAS NOT INDUCED.

\begin{tabular}{|c|c|c|c|c|c|c|c|c|c|}
\hline \multirow{2}{*}{$\dot{0}$} & \multirow{2}{*}{ Reported by. } & \multirow{2}{*}{ Age. } & \multirow{2}{*}{ 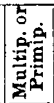 } & \multicolumn{2}{|c|}{ Vomiting. } & \multirow{2}{*}{ Treatment. } & \multirow{2}{*}{$\begin{array}{c}\text { Termi- } \\
\text { nation. }\end{array}$} & \multirow{2}{*}{ Condition and Remarks. } & \multirow{2}{*}{ Reference. } \\
\hline & & & & Begau. & Ended. & & & & \\
\hline $\boldsymbol{1}$ & $\begin{array}{l}\text { F. Minot, M.D. } \\
\text { Boston, Mass. }\end{array}$ & Young. & $\mathbf{M}_{2}$ & 8th week. & 20 th wk. & Nutritive enemata. & Recov. & $\begin{array}{l}\text { Vomiting was soon accompanied } \\
\text { by excessive salivation. "Pulse } \\
\text { gradually rose to upwards of } \\
130 \text { in the minute." There was } \\
\text { " much emaciation, prostra- } \\
\text { tion, loss of sleep, and depres- } \\
\text { sion of spirits." } \\
\text { ment, even an accidental loud } \\
\text { noise in the room would cause } \\
\text { urgent retching and rejection } \\
\text { of anything that happened to } \\
\text { be in the stomsch." Quicken- } \\
\text { ing May } 22 \text {; permanent relief } \\
\text { June 1. }\end{array}$ & $\begin{array}{l}\text { Boston Medical } \\
\text { and Surgical } \\
\text { Journal 1880, } \\
\text { cil. p. } 302 . \\
\\
\end{array}$ \\
\hline
\end{tabular}

Arch. Med., N. Y., 1880, iii, p. 269, 276.

S Murphy's Mid wifery, 2 d Edition, p. 94

- Science and Art of Midwifery, p. 120.

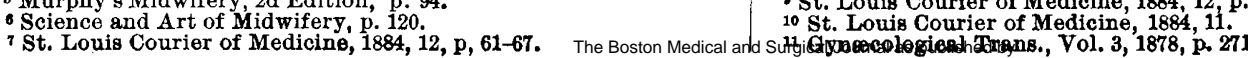

The New England Journal of Medicine. Downloaded from nejm.org at TULANE UNIV on June 22,2016 For personal use only. No other uses without permission. From the NEJM Archive. Copyright @ 2010 Massachusetts Medical Society.
8 Med. Rec., N. Y., 1885, Xxvii, p. 250.
${ }^{2}$ St. Louis Courier of Medicine, 1884, 12, p. 61, 67. 


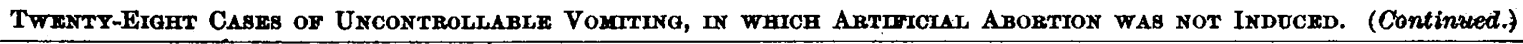

\begin{tabular}{|c|c|c|c|c|c|c|c|c|c|}
\hline \multirow{2}{*}{$\dot{0}$} & \multirow{2}{*}{ Reported by. } & \multirow{2}{*}{ Age. } & \multirow{2}{*}{ 苞兽 } & \multicolumn{2}{|c|}{ Vomiting. } & \multirow{2}{*}{ Treatment. } & \multirow{2}{*}{$\begin{array}{l}\text { Termi- } \\
\text { nation. }\end{array}$} & \multirow{2}{*}{ Condition and Remarks. } & \multirow{2}{*}{ Reference. } \\
\hline & & & & Began. & Ended. & & & & \\
\hline 2 & F. Copeman, M.D. & 26 & $\mathbf{P}$ & 6th mo. & $?$ & $\begin{array}{l}\text { Internsl remedies } \\
\text { failed. Rectal ali- } \\
\text { mentstion for } 3 \\
\text { weeks. Cervix di- } \\
\text { lated (Copeman's } \\
\text { method). }\end{array}$ & Recov. & $\begin{array}{l}\text { "Vomiting and retohing became } \\
\text { almost incessant." Vaginal } \\
\text { examination found posterior lip } \\
\text { of cervix hard and unyielding. } \\
\text { "Effect (Copeman's method) } \\
\text { was instantaneous, the stomach } \\
\text { retaining the first thing admin- } \\
\text { istered." }\end{array}$ & $\begin{array}{l}\text { British M. J., } \\
\text { I36. } \\
\\
\end{array}$ \\
\hline 3 & J. Farrant Fry, M. D., & $?$ & $\overline{\mathbf{M}_{0}}$ & 6th week. & 8th week. & $\begin{array}{l}\text { Internal treatment } \\
\text { failed. Cervix di- } \\
\text { lated (Copeman's } \\
\text { method). }\end{array}$ & Recov. & $\begin{array}{l}\text { The effect (Copernan's method) } \\
\text { was almost immediate; food } \\
\text { was retained and patientquick- } \\
\text { ly regained strength. Went to } \\
\text { term. }\end{array}$ & $\begin{array}{l}\text { British M. J., } \\
\text { Lond., 1880, i, } \\
\text { p. 399. }\end{array}$ \\
\hline 4 & $\begin{array}{l}\text { J. F. Baldwin, M. D., Co- } \\
\text { lumbus, Ohio. }\end{array}$ & 29 & $\mathbf{P}$ & 6th week. & 10th wk. & $\begin{array}{l}\text { Internal remedies } \\
\text { with failure. Cer- } \\
\text { vix dilated (Cope- } \\
\text { man's method). }\end{array}$ & $\overline{\mathbf{R e c o v}}$ & $\begin{array}{l}\text { "Nitrate of silver to erosion, } \\
\text { gave no relief." After dilata- } \\
\text { tion of cervix " all vomiting } \\
\text { ceased as by magic." }\end{array}$ & $\begin{array}{l}\text { Ohio Medical } \\
\text { Reoord, Co- } \\
\text { lumbus, 1879, } \\
\text { 1880, iv., p. } \\
\text { 198-200. }\end{array}$ \\
\hline 5 & $\begin{array}{l}\text { F. W. Lester, M.D., Key } \\
\text { West, Fla. }\end{array}$ & 30 & $\overline{\mathbf{M}}$ & $\begin{array}{c}\text { Almost } \\
\text { from time } \\
\text { of concep- } \\
\text { tion. }\end{array}$ & 14th wk. & $\begin{array}{l}\text { Internal treatment } \\
\text { failed. Ether to } \\
\text { pneumo-gas tric } \\
\text { nerves under ster- } \\
\text { no mastoids, al- } \\
\text { ternately. }\end{array}$ & Recov. & $\begin{array}{l}\text { For four weeks previous to ether } \\
\text { treatment, patient vomited } \\
\text { every few moments. Ether was } \\
\text { applied every two hours the } \\
\text { first day for ten minutes at } \\
\text { a time. In three days vomit- } \\
\text { ing ceased entirely. }\end{array}$ & $\begin{array}{l}\text { Med. Record, } \\
\text { N. Y. 1880, } \\
\text { Xviil. 474. } \\
\\
\end{array}$ \\
\hline 6 & $\begin{array}{l}\text { Jesse Crounse, M. D., } \\
\text { Knowersville. }\end{array}$ & 34 & $\overline{\mathbf{M}_{4}}$ & $\begin{array}{l}\text { Soon } \\
\text { after con- } \\
\text { ception. }\end{array}$ & $\begin{array}{l}\text { After 6th } \\
\text { month. }\end{array}$ & $\begin{array}{l}\text { Internal remedies } \\
\text { fai led. M or a l } \\
\text { treatment: slight } \\
\text { irritation of os, } \\
\text { pretending at- } \\
\text { tempted abortion } \\
\text { which patient de- } \\
\text { si red. "E a ch } \\
\text { remedy was in } \\
\text { turn given a trial } \\
\text { and still no im- } \\
\text { provemen t, b ut } \\
\text { on the contrary I } \\
\text { saw her wasting } \\
\text { and be o m ing } \\
\text { more feeble every } \\
\text { day." }\end{array}$ & Recov. & $\begin{array}{l}\text { With flrst child suffered from } \\
\text { nausea and romiting for seven } \\
\text { and one-half months. In second } \\
\text { pregnancy, labor was induced } \\
\text { at three and one-half months, } \\
\text { successfully. In third, labor } \\
\text { was again induced at third } \\
\text { month without any trouble. } \\
\text { In this case, patient begged } \\
\text { physician to induce labor, and } \\
\text { finally he consented. He, how- } \\
\text { ever, merely irritated os with } \\
\text { a probe, she thinking an abor- } \\
\text { tion had been performed. Vom- } \\
\text { iting ceased and on return of } \\
\text { same was soon relieved by same } \\
\text { method. No vomiting after } \\
\text { sixth month. Patient was de- } \\
\text { livered at term. }\end{array}$ & $\begin{array}{l}\text { Med. Annals, } \\
\text { Albany, 1882, } \\
\text { iii. p. 226-228. } \\
\text { f } \\
\text { f } \\
\text { f } \\
\text { r }\end{array}$ \\
\hline 7 & $\begin{array}{l}\text { T. C. Wallace, M.D., Cam- } \\
\text { bridge, N.Y. }\end{array}$ & $?$ & $\overline{\mathbf{P}}$ & 8th week. & 13th wk. & $\begin{array}{l}\text { Everything inter- } \\
\text { nally. Copeman's } \\
\text { method. Nitrate } \\
\text { of silver (solid) to } \\
\text { cervix. Relieved } \\
\text { by pop-corn. }\end{array}$ & Recov. & $\begin{array}{l}\text { "Conflned to bed for more than } \\
\text { four weeks, pale, languid, } \\
\text { pulse } 120 \text { and weak, and so pros- } \\
\text { trated as to be unable to raise } \\
\text { head from pillow. For the } \\
\text { past sixteen days her stomach } \\
\text { has absolutely refused to retain } \\
\text { anything ; everything, food, } \\
\text { medioine, even a swallow of } \\
\text { water being instantly rejected." } \\
\text { Rectal alimentation only for } \\
\text { past six days. Immediate re- } \\
\text { lief after pop-corn. }\end{array}$ & $\begin{array}{l}\text { Med. and Sur. } \\
\text { Rep., Phila., } \\
\text { 1883, xlviii. p. } \\
\text { 172. } \\
\\
\\
\\
\\
\end{array}$ \\
\hline 8 & John N. Upshur, M.D. & 24 & $\mathbf{P}$ & 7 th week. & 10th wk. & $\begin{array}{l}\text { Internal treatment } \\
\text { failed. Relieved } \\
\text { by Hodge's dou- } \\
\text { ble lever pessary } \\
\text { (closed). }\end{array}$ & Recov. & $\begin{array}{l}\text { Retroversion discovered on 10th } \\
\text { week. Relieved by pessary. } \\
\text { Previous to insertion of pessary, } \\
\text { patient's condition became } \\
\text { steadily worse, she "becoming } \\
\text { more prostrated and her dis. } \\
\text { gust of food increasing daily." }\end{array}$ & $\begin{array}{l}\text { Am. J. Obst., } \\
\text { N. Y., 1884, } \\
\text { Xvii. p. 916, } \\
917 .\end{array}$ \\
\hline 9 & J. W. Ayle, M.D. & $?$ & $\overline{\mathbf{M}}$ & $2 \mathrm{~d}$ month. & $\begin{array}{l}\text { 2d month, } \\
\text { 2d week. }\end{array}$ & $\begin{array}{l}\text { Relieved by Hors- } \\
\text { ford's Acid Phos- } \\
\text { phate and chick- } \\
\text { en-water, other } \\
\text { internal remedies } \\
\text { having failed. }\end{array}$ & Recov. & $\begin{array}{l}\text { "Nausea and vomiting, ceased." } \\
\text { "Acted like a charm." }\end{array}$ & $\begin{array}{l}\text { Med. Sur. Rep., } \\
\text { Phila., 1884, } \\
\text { p. 757. }\end{array}$ \\
\hline 10 & Dr. Prewitt. & $\overline{\text { Young. }}$ & $?$ & $\begin{array}{l}\text { About 2d } \\
\text { month. }\end{array}$ & $?$ & $\begin{array}{l}\text { Morphine subcuta- } \\
\text { neously. }\end{array}$ & Recov. & $\begin{array}{l}\text { "Not a particle of food for a } \\
\text { week" previous to morphine } \\
\text { treatment. Patient was kept } \\
\text { under the influence of mor- } \\
\text { phine all the time. "Takes } \\
\text { none now, though she did get } \\
\text { somewhat of the habit." }\end{array}$ & $\begin{array}{l}\text { St. Bartholo- } \\
\text { mew's Hosp. } \\
\text { Rep., Lond., } \\
\text { 1883, p. 257. } \\
\text { t. }\end{array}$ \\
\hline$\overline{11}$ & $\begin{array}{l}\text { C. E. Stedman, M.D., Bos- } \\
\text { ton, Mass. }\end{array}$ & Young. & $\overline{\mathbf{M}_{2}}$ & The Boston Me & 14th wk. & $\begin{array}{l}\text { Everything inter- } \\
\text { nally. Etherial } \\
\text { tinct. of iodine } \\
\text { to cervix. Rectal } \\
\text { alimentation. }\end{array}$ & Recov. & $\begin{array}{l}\text { First labor normal. " When I } \\
\text { visited her remedies prescribed } \\
\text { had lost effect, and she was in } \\
\text { an alarming condition ; every } \\
\text { particle of food had been re-- } \\
\text { jected for forty-eight hours. } \\
\text { Patient had headache, fleeting } \\
\text { delirium, pinched countenance, } \\
\text { dry brown tonge, constant } \\
\text { nausea, little sleep, epigastric } \\
\text { and abdominal pain, vomiting } \\
\text { up everything, and rapid pulse. } \\
\text { The os was patulous and very } \\
\text { red. Cervix was flexed and } \\
\text { very soft. External Tr. Iodi } \\
\text { applied to cervix liberally. } \\
\text { Vomiting ceased in twenty- } \\
\text { four hours. Pattent vomitted } \\
\text { only twioe after that time." }\end{array}$ & $\begin{array}{l}\text { Boston Med. } \\
\text { and Sur. J., } \\
\text { 1883, 109, p. } \\
\text { 529. } \\
\end{array}$ \\
\hline
\end{tabular}




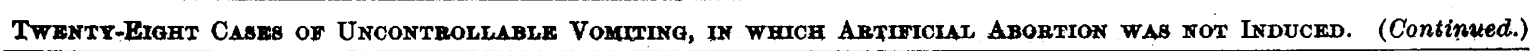

\begin{tabular}{|c|c|c|c|c|c|c|c|c|c|}
\hline \multirow{2}{*}{$\dot{0}$} & \multirow{2}{*}{ Reported by. } & \multirow{2}{*}{ Age. } & \multirow{2}{*}{ 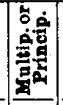 } & \multicolumn{2}{|c|}{ Vomiting, } & \multirow{2}{*}{ Treatment. } & \multirow{2}{*}{$\begin{array}{l}\text { Termi- } \\
\text { nation. }\end{array}$} & \multirow{2}{*}{ Condition and Remarks. } & \multirow{2}{*}{ Reference. } \\
\hline & & & & Began. & Fnded. & & & & \\
\hline 12 & $\begin{array}{l}\text { Fenry F. Campbell, M.D., } \\
\text { Augusta, Ga. }\end{array}$ & 29 & $\mathbf{M}_{3}$ & $?$ & $\begin{array}{c}\text { About } \\
\text { 16th wk. }\end{array}$ & $\begin{array}{l}\text { Everything inter- } \\
\text { nally. Nitrate of } \\
\text { silver (solid) to } \\
\text { cervix. Rectai ali- } \\
\text { mentation alone } \\
\text { for } 52 \text { days }\end{array}$ & Recov. & $\begin{array}{l}\text { Patient aborted at three and } \\
\text { one-half months. Foetus dead. } \\
\text { Nausea did not cease for over } \\
\text { two weeks, during which time } \\
\text { she was nourished solely by } \\
\text { enemata and for a much longer } \\
\text { time this " had to be continued } \\
\text { as supplementary to her very } \\
\text { gradually returning power to } \\
\text { take and retain food by gastric } \\
\text { ingestion." }\end{array}$ & $\begin{array}{l}\text { Gynacological } \\
\text { Transactions, } \\
1878 \text {, iii, 268 - } \\
298 \text {. }\end{array}$ \\
\hline$\overline{\mathbf{1 3}}$ & Henry K. Leake, M.D. & $?$ & $?$ & pth week. & 12th wk. & $\begin{array}{l}\text { Everything inter- } \\
\text { nally. Ce rvi } \\
\text { thoroughly dilat- } \\
\text { ed. }\end{array}$ & Rocov. & $\begin{array}{l}\text { Labor set in (probably due to } \\
\text { dilatation of cervix) about the } \\
\text { 12th week. " Patient recovered } \\
\text { after a slow and painful con- } \\
\text { valescence from a pelvic cel- } \\
\text { lulitis, which supervened on } \\
\text { the expulsion of the foetus. Im- } \\
\text { mediate relief to vomiting after } \\
\text { abortion." }\end{array}$ & $\begin{array}{l}\text { Med.Rec.,N.Y., } \\
1885, \text { xxvii, p. } \\
256 . \\
\end{array}$ \\
\hline 14 & W. J. Collins, M.D. & 38 & $\overline{\mathbf{M}_{7}}$ & $4 \mathrm{mos}$. & $6 \frac{1}{2} \mathrm{mos}$. & $\begin{array}{l}\text { Internal remedies } \\
\text { only. }\end{array}$ & Recov. & $\begin{array}{l}\text { Patient aborted at six and one- } \\
\text { half months. Labors natural } \\
\text { before. }\end{array}$ & $\begin{array}{l}\text { St. Bartholo- } \\
\text { mew's Hosp. } \\
\text { Rep., Lond., } \\
\text { 1883, p. 125. }\end{array}$ \\
\hline$\overline{15}$ & Dr. Murphy, London. & $?$ & $\overline{\mathbf{P}}$ & $\begin{array}{l}\text { Almost } \\
\text { from time } \\
\text { of concep- } \\
\text { tion. }\end{array}$ & $\begin{array}{c}\text { About 3d } \\
\text { month. }\end{array}$ & $\begin{array}{l}\text { Internal remedies } \\
\text { only. }\end{array}$ & Desth. & $\begin{array}{l}\text { "About the third month it (the } \\
\text { romiting) became so severe as } \\
\text { to cause great emaciation } \\
\text { and exhaustion." Several reme- } \\
\text { dies were used to no purpose, } \\
\text { and at the eleventh hour the } \\
\text { question was asked:-"."What } \\
\text { further could be done?" She } \\
\text { was fast sinking ; the time was } \\
\text { past to attempt the induction } \\
\text { of permature labor and she } \\
\text { died in about two days after- } \\
\text { wards." }\end{array}$ & $\begin{array}{l}\text { Murphy's Mid- } \\
\text { wifery,2d ed., } \\
\text { p. 94. } \\
\end{array}$ \\
\hline$\overline{\mathbf{1 6}}$ & Dr. Haighton. & $?$ & $\overline{\mathbf{M}_{4}}$ & $\begin{array}{l}\text { About 6th } \\
\text { month be- } \\
\text { gan to } \\
\text { grow } \\
\text { worse. }\end{array}$ & $\begin{array}{c}\text { Probably } \\
\text { about 7t: } \\
\text { months. }\end{array}$ & $\begin{array}{l}\text { Internal remedies } \\
\text { only. }\end{array}$ & Death. & $\begin{array}{l}\text { "She had most obstinate vomit- } \\
\text { ing in two previous pregnan- } \\
\text { cies." Siokness would gome- } \\
\text { times intermit. Slight im- } \\
\text { provement caused the doctor } \\
\text { to defer artifial abortion. The } \\
\text { next day she was taken ex- } \\
\text { ceedingiy ill and died in two } \\
\text { days. }\end{array}$ & $\begin{array}{l}\text { Obst. Med., vol. } \\
\text { if. p. 871. }\end{array}$ \\
\hline 17. & Dr. Johnson. & 30 & $\mathbf{P}$ & $?$ & 4th mo. & $\begin{array}{l}\text { Internsl remedies } \\
\text { only. }\end{array}$ & Death. & $\begin{array}{l}\text { "Pregnancy was not detected } \\
\text { but the disease was attributed } \\
\text { to the pylorus." "The sick- } \\
\text { ness (vomiting) and emaciation } \\
\text { were the only symptoms pre- } \\
\text { sent." "She died, and after } \\
\text { death no morbid appearances } \\
\text { were observable in any part of } \\
\text { the body. The uterus contained } \\
\text { a fotus about four months old. } \\
\text { This patient was literally } \\
\text { starved to death." }\end{array}$ & $\begin{array}{l}\text { Lancet, Lond., } \\
\text { Mar. 3, 1838. } \\
\text { p. 825. } \\
\end{array}$ \\
\hline 18 & $\begin{array}{l}\text { J. Marion Sims, M.D., N. } \\
\text { Y. }\end{array}$ & Young. & $\mathbf{P}$ & $\overline{2 d \text { week. }}$ & $?$ & $\begin{array}{c}\text { Internal remedies } \\
\text { only.| }\end{array}$ & Death. & $\begin{array}{l}\text { Artiflcial abortion advised and } \\
\text { refused. Died of heart clot } \\
\text { started up " by getting out of } \\
\text { bed to answer a call of nature." }\end{array}$ & $\begin{array}{l}\overline{\text { Arch. Medical }} \\
\text { N. Y., } 1880 \text {, } \\
\text { iii. } 269-276 .\end{array}$ \\
\hline$\overline{19}$ & $\begin{array}{l}\text { Geo. Davenport, M.D., E. } \\
\text { Randolph, Vt. }\end{array}$ & 28 & $\mathbf{P}$ & $?$ & $?$ & Internally only. & Desth. & $\begin{array}{l}\text { Married about one year. At- } \\
\text { tacked with nausea and vomit- } \\
\text { ing and died in five } \\
\text { weeks in spite of all treat- } \\
\text { ment. Pregnancy was not } \\
\text { recognized, the diagnosis being } \\
\text { a low form of bilious vomiting, } \\
\text { great emaciation. Pregnancy } \\
\text { conflrmed by autopsy. }\end{array}$ & $\begin{array}{l}\text { Tr.Ver. M. Soc. } \\
1878, \text { St. Al- } \\
\text { bang, 1879, p. } \\
39,40 . \\
\end{array}$ \\
\hline 20 & $\begin{array}{l}\text { Geo. Davenport, M.D., E. } \\
\text { Randolph, vt. }\end{array}$ & Young. & $\overline{\mathbf{P} ?}$ & bth week. & 12th wk. & 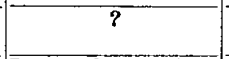 & Death. & "She died of exhaustion." & $\overline{\text { (Same as above) }}$ \\
\hline $\mathbf{8 1}$ & $\begin{array}{l}\text { Geo. Davenport, M.D., E. } \\
\text { Randolph, Vt. }\end{array}$ & $\begin{array}{l}\text { Married } \\
\text { several } \\
\text { years. ? }\end{array}$ & $?$ & $?$ & $?$ & $\begin{array}{l}\text { Internal remedies. } \\
\text { Morphine per rec- } \\
\text { tum. }\end{array}$ & Death. & 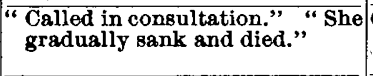 & (Sameas above) \\
\hline 8 & $\begin{array}{l}\text { W.S. Brown, M.D., Stone- } \\
\text { ham. }\end{array}$ & 26 & $\overline{\mathbf{M}_{2}}$ & $\begin{array}{l}\text { About the } \\
\text { 12th wk. }\end{array}$ & 16th wk. & $\begin{array}{c}\text { Interna } 11 \text { y o n l y. } \\
\text { Usual remedies } \\
\text { had no effect. }\end{array}$ & Death. & $\begin{array}{l}\text { "Labor pains set in on October } \\
\text { 14th, about } 2 \text { A. M., and the } \\
\text { fetus was expelled at 7 A. M." } \\
\text { "Convulsions and coma super- } \\
\text { vened and terminated "the } \\
\text { scene about 9 A. M." "One } \\
\text { thing remarkable was the } \\
\text { absence of emaciation." The } \\
\text { question occurs "What was } \\
\text { the cause of death?" "It was } \\
\text { not due to starvation, nor to } \\
\text { Bright's disease, nor to any } \\
\text { operation upon the womb. } \\
\text { Exhaustion of nervous energy, } \\
\text { therefore, seems the most prob- } \\
\text { able answer." }\end{array}$ & $\begin{array}{l}\text { Jour. of the } \\
\text { Gynæc olog i- } \\
\text { cal Soc. of } \\
\text { Boston, vol. } \\
\text { ii. p. 208. } \\
\\
\end{array}$ \\
\hline $\bar{A}$ & A. H. P. Louf, M.D. & 22 & $\overline{\mathbf{P Q}}$ & $\begin{array}{l}\text { Probably } \\
\text { 6th to } 8 \text { th } \\
\text { week. }\end{array}$ & $\begin{array}{l}\text { About } \\
\text { 12th wk. }\end{array}$ & $\begin{array}{l}\text { Internal ly on ly. } \\
\text { Pregna n oy not } \\
\text { Buspected. }\end{array}$ & Death. & $\begin{array}{l}\text { The patient was not married. } \\
\text { Pregnancy was not suspected. } \\
\text { "The case was mistaken for gas- } \\
\text { tric ulcer." "The symptoms } \\
\text { also pointed to a local gastric }\end{array}$ & $\begin{array}{l}\text { Proc. Med. Soc. } \\
\text { County Kings } \\
\text { Brook'n 1883, } \\
\text { 1834, viii. } 48 \text { - } \\
\text { 50. }\end{array}$ \\
\hline
\end{tabular}




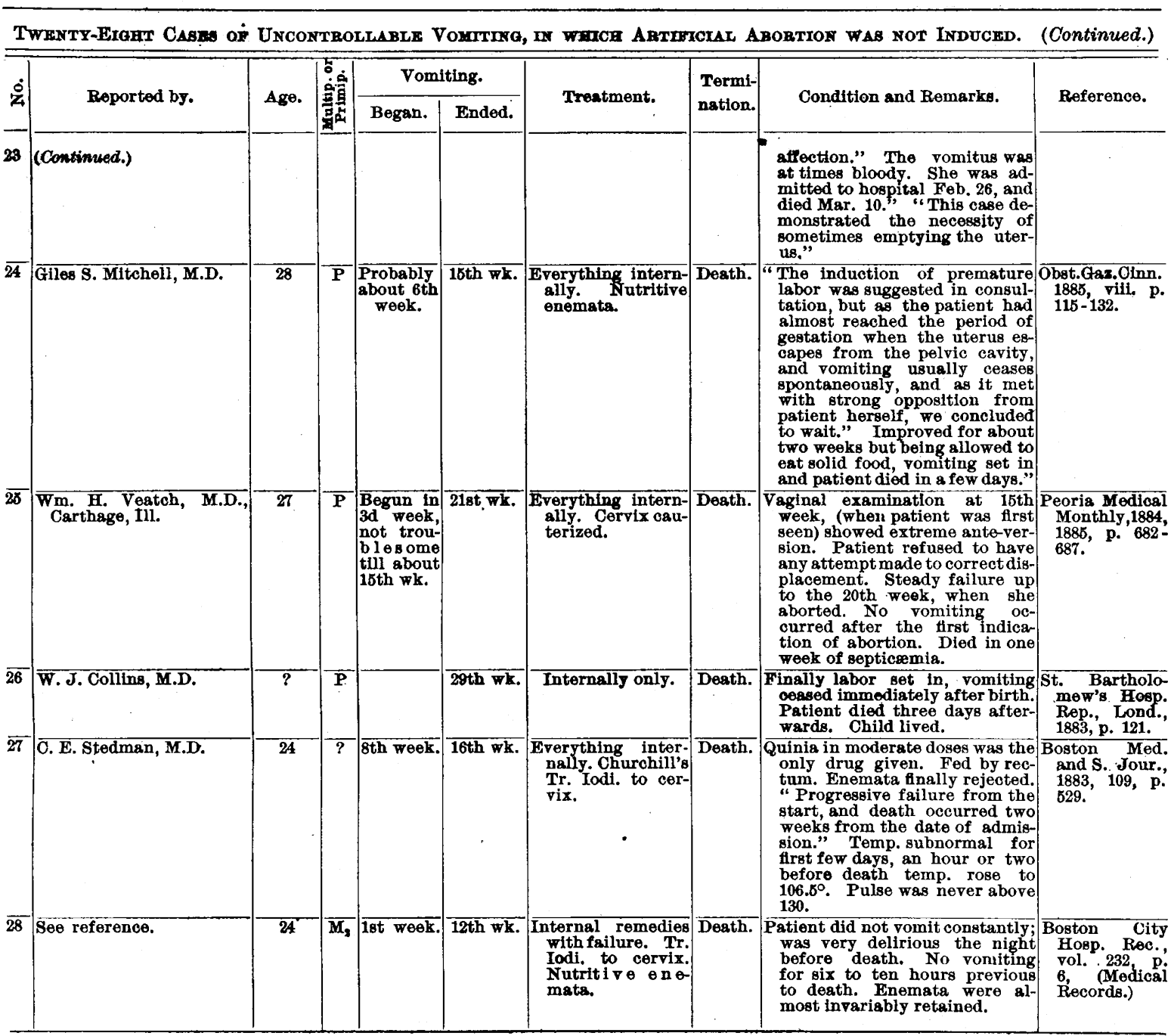

SUMMARY.

Recoveries $\left\{\begin{array}{l}\text { Multip. } \\ \text { Primip. }\end{array}\right.$ $\left\{\begin{array}{l}\text { Primip. } \\ \text { Not atated } 2\end{array}\right.$ Spontaneous abortion with recovery 


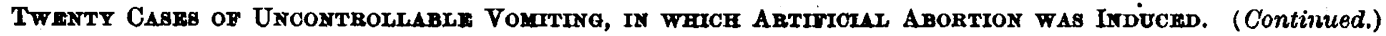

\begin{tabular}{|c|c|c|c|c|c|c|c|c|c|}
\hline \multirow{3}{*}{$\begin{array}{l}\dot{2} \\
\mathbf{Z} \\
\end{array}$} & \multirow{3}{*}{$\begin{array}{r}\text { Reported by. } \\
\text { W. J. Collins, M.D. }\end{array}$} & \multirow{3}{*}{$\frac{\text { Age. }}{26}$} & \multirow{3}{*}{ 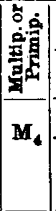 } & \multicolumn{2}{|c|}{ Vomiting. } & \multirow{2}{*}{ Trestment. } & \multirow{3}{*}{$\begin{array}{c}\text { Termi- } \\
\text { nation. }\end{array}$} & \multirow{2}{*}{ Condition and Remarks. } & \multirow{2}{*}{ Reference. } \\
\hline & & & & Began. & Ended. & & & & \\
\hline & & & & $\underset{\text { month. }}{\text { mbout 6th }}$ & $?$ & $\begin{array}{l}\text { Introduction of a } \\
\text { gum elastic cath- } \\
\text { eter } 8 \text { inches. }\end{array}$ & & $\begin{array}{l}\text { " Vomiting ceased immediately } \\
\text { after birth." }\end{array}$ & $\begin{array}{l}\text { St. Bartholo- } \\
\text { mew's Hosp. } \\
\text { Rep., Lond., } \\
\text { 1883, p. 123. }\end{array}$ \\
\hline 4 & $\begin{array}{l}\text { S. G. Moses, M.D., St. } \\
\text { Louis, Mo. }\end{array}$ & $\begin{array}{c}\text { Under } \\
.30 \\
\text { years. } \\
\end{array}$ & $\overline{\mathbf{M}}$ & $?$ & $\begin{array}{l}\text { Between } \\
\text { 2d and } 3 d \\
\text { month. }\end{array}$ & $\begin{array}{l}\begin{array}{c}\text { Bougie to fundus } \\
\text { between } \\
\text { branes. }\end{array} \\
\end{array}$ & Recov. & $\begin{array}{l}\text { Never vomited after introduc- } \\
\text { toon of bougie and eventually } \\
\text { aborted." }\end{array}$ & $\begin{array}{l}\text { St. Louis Cour- } \\
\text { ier of Med., } \\
1884,11, \text { p. 252. }\end{array}$ \\
\hline$\overline{5}$ & S. G. Moses, M.D. & $\begin{array}{l}\text { Under } \\
30 \\
\text { years. }\end{array}$ & $\overline{M_{2}}$ & $?$ & $\begin{array}{l}\text { Between } \\
2 \mathrm{~d} \text { and 3d } \\
\text { month. }\end{array}$ & Orum punctured. & Recov. & $\begin{array}{l}\text { When first seen was in mori- } \\
\text { bund condition." Out of danger } \\
\text { in twenty-four hours. In first } \\
\text { pregnancy vomited several } \\
\text { weeks and then aborted, with } \\
\text { relief from vomiting. Several } \\
\text { children since without trouble. }\end{array}$ & $\begin{array}{l}\text { St. Louis Cour- } \\
\text { ier of Med., } \\
1884,11,250- \\
262 . \\
\end{array}$ \\
\hline 6 & $\begin{array}{l}\text { R. Scot-Skining, M.B. et } \\
\text { Ch. m., Edin, Phys. to } \\
\text { the Prince Alfred Hos- } \\
\text { pital, Sydney. }\end{array}$ & 21 & $?$ & $3 \mathbf{m o}$ & $\begin{array}{l}\text { About } \\
\text { ith mo. }\end{array}$ & $\begin{array}{l}\text { All the usual medi- } \\
\text { cinal rem ed i es } \\
\text { were exhausted. } \\
\text { Finally, introduc- } \\
\text { tion of bougie and } \\
\text { dilatation of cer- } \\
\text { vix with Barnes' } \\
\text { fluid dilators. }\end{array}$ & Recov. & $\begin{array}{l}\text { Patient was a passenger on board } \\
\text { an emigrant ship, sailing from } \\
\text { Plymouth, Eng., to Australia. } \\
\text { "The morning sickness was } \\
\text { intensifled by sea-sickness." } \\
\text { Bass Ale was retained when } \\
\text { all else was immediately vom- } \\
\text { ited. "For the first few days } \\
\text { after delivery the patient re- } \\
\text { mained in a very low state, but } \\
\text { with the entire absence of the } \\
\text { distressing vomiting which had } \\
\text { before existed. Food was now } \\
\text { readily retained, and in an as- } \\
\text { tonishing short time I had the } \\
\text { satisfaction of seeing her in a } \\
\text { condition which removed all } \\
\text { further anxiety from my } \\
\text { mind." }\end{array}$ & $\begin{array}{l}\text { The Australa- } \\
\text { sian Medical } \\
\text { Gazette,Sept. } \\
\text { 1884, vol. fil. } \\
\text { No. 12. }\end{array}$ \\
\hline$\overline{7}$ & Dr. Scott. & $\begin{array}{c}\text { ? } \\
\text { Probs- } \\
\text { bly } \\
\text { young. }\end{array}$ & $\mathbf{P}$ & 6th week. & 10th wk. & $\begin{array}{l}\text { Artiflcial abortion. } \\
\text { Method not stated. }\end{array}$ & Recov. & $\begin{array}{l}\text { "Nausea left from time fotus } \\
\text { came away." }\end{array}$ & $\begin{array}{l}\text { St. Louis Cour- } \\
\text { ier of Med., } \\
1884,12 \text {, p. } 61= \\
67 .\end{array}$ \\
\hline$\overline{8}$ & Dr. Coles. & $?$ & $?$ & $?$ & $?$ & $\begin{array}{l}\text { Artifioial abortion. } \\
\text { Method not stated. }\end{array}$ & Recov. & $\begin{array}{l}\text { Patient was relieved from nau- } \\
\text { sea and vomiting instantly. }\end{array}$ & $\begin{array}{l}\text { St. Louis Cour. } \\
\text { of Med., 1884, } \\
11, \text { p. 259-260. }\end{array}$ \\
\hline 9 & Dr. Ward. & 38 & $\overline{\mathbf{P}}$ & $?$ & 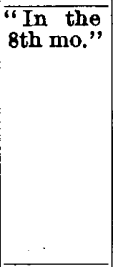 & $\begin{array}{l}\text { Everything inter- } \\
\text { nally failed. In. } \\
\text { duced abortion. } \\
\text { Hot douche to in- } \\
\text { ternal os followed } \\
\text { by Barnes' dila- } \\
\text { tor. }\end{array}$ & Recov. & $\begin{array}{l}\text { Patient was seen " on account of } \\
\text { such excessive prostration due } \\
\text { to continuous vomiting." } \\
\text { "Every therapentic means } \\
\text { had been adopted without } \\
\text { avail." Podalic version. Dead } \\
\text { hydrocephalic child with peel- } \\
\text { ing epidermis. "In less than } \\
\text { twenty-four hours she was able } \\
\text { to retain whatever nourish- } \\
\text { ment was given." Rapid re- } \\
\text { covery. }\end{array}$ & $\begin{array}{l}\text { N. } \overline{\mathbf{Y}} \text {. Med. J., } \\
1880 \text {, xxxi. p. } \\
406 .\end{array}$ \\
\hline$\overline{10}$ & $\begin{array}{l}\text { Wm. Warren Potter,M.D., } \\
\text { Batavia, N.X. }\end{array}$ & 23 & $\mathbf{P}$ & $\begin{array}{c}\text { ? } \\
\text { Probsbly } \\
\text { about10th } \\
\text { week. }\end{array}$ & 14th wk. & $\begin{array}{l}\text { Internal remedies } \\
\text { failed. Nitrate of } \\
\text { silver. T T in ct. } \\
\text { lodi. locally fail- } \\
\text { ed. A r t i t c i al } \\
\text { abortion. }\end{array}$ & Recov. & $\begin{array}{l}\text { "There was inter-current catar- } \\
\text { rhal bronchitis present." "Rec- } \\
\text { tal alimentation and medica- } \\
\text { tion having failed to arrest the } \\
\text { progress of malady, the direful } \\
\text { alternative of putting an end } \\
\text { to the pregnancy was forced } \\
\text { upon me." }\end{array}$ & 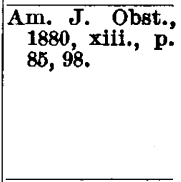 \\
\hline$\overline{11}$ & M. H. Jordan, M.D. & 24 & $\mathbf{P}$ & $?$ & 14th wk. & $\begin{array}{l}\text { Internal treatment } \\
\text { fadled. Nitrate of } \\
\text { silver to cervix } \\
\text { failed. Artifleial } \\
\text { abortion. Cervix } \\
\text { dilated and foetus } \\
\text { scooped out by } \\
\text { hand. }\end{array}$ & Recov. & $\begin{array}{l}\text { "When first seen, patient had } \\
\text { been contined to bed for five } \\
\text { weeks with a distressing and } \\
\text { uncontrollable nausea and } \\
\text { vomiting." She was " much } \\
\text { emaciated, quick pulse, some } \\
\text { fever, and retained compars- } \\
\text { tively nothing on her stomach." } \\
\text { There was considerable diff- } \\
\text { culty in bringing on labor pains } \\
\text { but finally it was successfully } \\
\text { accomplished. Patient was so } \\
\text { weak at time of abortion, that } \\
\text { she could not turn herself in } \\
\text { bed. "After the operation } \\
\text { she never became sick at the } \\
\text { stomach or vomited a single } \\
\text { time." In two weeks she was } \\
\text { able to be carried home from } \\
\text { Birmingham, Ala., to chatta- } \\
\text { nooga, Tenn. "If this woman } \\
\text { had not have aborted, she cer- } \\
\text { tainly would have died." } \\
\end{array}$ & $\begin{array}{r}\text { Ameri'n Pract., } \\
\text { Louis ville, } \\
\text { 1879, xx. 305 - } \\
309 . \\
.\end{array}$ \\
\hline$\overline{12}$ & $\begin{array}{l}\text { W. T. Wright, M.D., Wtn- } \\
\text { fleld, Kansas. }\end{array}$ & 24 & $\mathbf{P}$ & $\begin{array}{c}\text { 6th or 7th } \\
\text { week. } \\
.\end{array}$ & $\begin{array}{l}\text { About } \\
\text { 10th wk. } \\
\text { Thirte en } \\
\text { days after } \\
\text { s or io u s } \\
\text { symptoms } \\
\text { set in. }\end{array}$ & $\begin{array}{l}\text { Everything inter- } \\
\text { nally with fail- } \\
\text { ure. Ar t i f cisl } \\
\text { abortion. Pains } \\
\text { set up by electric- } \\
\text { ity, ergot sounds } \\
\text { and tents failing. }\end{array}$ & Recov. & $\begin{array}{l}\text { "Pulse gradually rose until it } \\
\text { reached } 140-150 \text { at time of } \\
\text { abortion, at which time the } \\
\text { following, conditions were } \\
\text { present:" "She was a fleshy } \\
\text { woman, now the skin hang8 } \\
\text { loose on her limbs, eyes deeply } \\
\text { sunken; a markedly pinched } \\
\text { appearance of her features. } \\
\text { Respirations 40 per minute, } \\
\text { pulse rapid and indlstinct, sur- } \\
\text { fao hot and dry, extreme dysp- } \\
\text { noa, restless throwing about } \\
\text { of limbs, general tremor. On } \\
\text { the day following the sbortion } \\
\text { she called for food which was } \\
\text { dd brelighed and retained. }\end{array}$ & $\begin{array}{l}\text { Peoria Medical } \\
\text { Month., 1883, } \\
\text { 4, iv., p. 6-14. } \\
\end{array}$ \\
\hline
\end{tabular}


Twhaty Cases of Uncontrolyable Vomiting, in whrch artipiclal Abortron was Indeced. (Continued.)

\begin{tabular}{|c|c|c|c|c|c|c|c|c|c|}
\hline \multirow{2}{*}{$\dot{\mathrm{z}}$} & \multirow{2}{*}{ Reported by. } & \multirow{2}{*}{ Age. } & \multirow{2}{*}{ 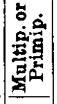 } & \multicolumn{2}{|c|}{ Vomiting. } & \multirow{2}{*}{ Treatment. } & \multirow{2}{*}{$\begin{array}{l}\text { Term1- } \\
\text { nation. }\end{array}$} & \multirow{2}{*}{ Condition and Remarks. } & \multirow{2}{*}{ Reference. } \\
\hline & & & & Began. & Ended. & & & & \\
\hline 13 & $\begin{array}{l}\text { T. G. Thoms, M.D., New } \\
\text { York. }\end{array}$ & $\stackrel{?}{?}$ & $\mathbf{P}$ & 3d week. & 10th wk. & $\begin{array}{c}\text { Artificial abortion. } \\
\text { Cervix dilated and } \\
\text { foetus and placen- } \\
\text { ta scooped out } \\
\text { with or din a r } \mathrm{y} \\
\text { wire curette. }\end{array}$ & Recov. & $\begin{array}{l}\text { Patient dispelled a " phantom" } \\
\text { tumor about three months be- } \\
\text { fore, thinking herself to be } \\
\text { pregnant. When first seen had } \\
\text { been vomiting two and one- } \\
\text { half months. As a result of } \\
\text { the vomiting she had become } \\
\text { excessirely emaciated." After } \\
\text { curetting the vomiting ceased } \\
\text { at once and patient recovered } \\
\text { entirely. }\end{array}$ & $\begin{array}{l}\text { Am. J. Obst., } \\
\text { p. } 674 . \\
\text { 1881, xdv. }\end{array}$ \\
\hline 14 & $\begin{array}{l}\text { N. C. B. Haviland, M.D., } \\
\text { Danvile, Vt. }\end{array}$ & 23 & $?$ & $\begin{array}{c}\text { 5th or } 6 \text { th } \\
\text { week. }\end{array}$ & 15th wk. & $\begin{array}{l}\text { Internal treatment } \\
\text { failed. Artifleisi } \\
\text { abortion by injec- } \\
\text { tion of tepid wa- } \\
\text { ter, one ounce, in } \\
\text { to uterine cavity. }\end{array}$ & Reoov. & 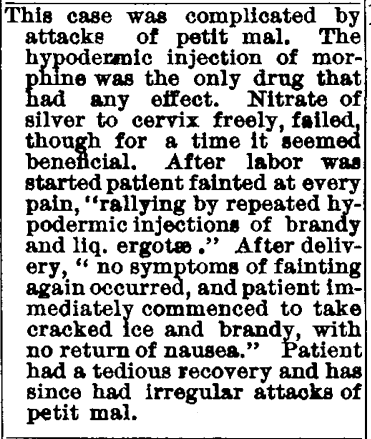 & $\begin{array}{r}\text { Am. J. Obst., } \\
\text { 1883, xvi. No. } \\
\text { 2, p. 165-168. } \\
\\
\end{array}$ \\
\hline 15 & $\begin{array}{l}\text { Geo. Davenport, M.D., E. } \\
\text { Randolph, Vt. }\end{array}$ & 34 & $\overline{\mathbf{M}_{5}}$ & th wk. ? & $\begin{array}{c}\text { 6th or 7th } \\
\text { week. }\end{array}$ & $\begin{array}{c}\text { Internal remedies } \\
\text { failed. Artificial } \\
\text { abortion- - sound } \\
\text { introduced. } \\
\end{array}$ & Rocov. & $\begin{array}{l}\text { No nourishment in stomach for } \\
\text { over two weeks. Life sus } \\
\text { tained solely by nutritive ene } \\
\text { mate. Pulse 1200130. "Could } \\
\text { not turn herself in bed," noor } \\
\text { move her lower limbs. Fea- } \\
\text { tures pale and sunken. Death } \\
\text { appeared inevitable. "The } \\
\text { patient, notwithstanding her } \\
\text { great prostration, made a good } \\
\text { recovery, slowly to be sure, but } \\
\text { with not one untoward symp- } \\
\text { tom." } \\
\end{array}$ & $\begin{array}{l}\text { Tr. Ver. M. Soc. } \\
\text { 1878, St. A1- } \\
\text { bans, 1879, 39, } \\
49 . \\
\\
\\
\end{array}$ \\
\hline 16 & See reference. & 20 & $\mathbf{P}$ & 5th wk. & $\begin{array}{c}\text { Abront } \\
17 \text { th wk. }\end{array}$ & $\begin{array}{l}\text { Internal remedies } \\
\text { failed. Tr. iodi. } \\
\text { to cervix. Artifi- } \\
\text { cial abortion by } \\
\text { means of sponge- } \\
\text { tent and sonnd } \\
\text { passed into uter- } \\
\text { ine cavity. }\end{array}$ & \begin{tabular}{|l|} 
Recov. \\
\\
\end{tabular} & 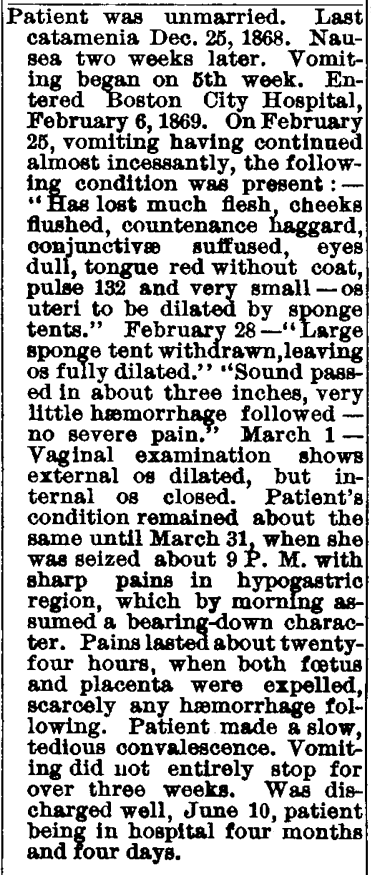 & $\begin{array}{l}\text { Boston City } \\
\text { Hosp. Med. } \\
\text { Records, vol. } \\
\text { 35, p. 132. } \\
\\
\end{array}$ \\
\hline$\overline{17}$ & $\begin{array}{l}\text { Geo. T. Elliot, M.D., New } \\
\text { York. }\end{array}$ & 32 & $\overline{\mathbf{M}_{0}}$ & $\begin{array}{l}\text { About 6th } \\
\text { month. }\end{array}$ & $\begin{array}{l}\text { Between } \\
7 \text { th and } \\
\text { 8th mo. }\end{array}$ & $\begin{array}{l}\text { Internally every- } \\
\text { thing failed. Ar- } \\
\text { tifloial abortion } \\
\text { by means of hot } \\
\text { douches. }\end{array}$ & Death. & 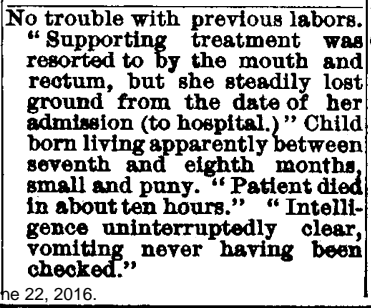 & $\begin{array}{l}\text { bst. Clin., p. } \\
\text { 167. }\end{array}$ \\
\hline
\end{tabular}


BOSTON MEDIOAL AND SURGICAL JOURNAL. [FEBRUARY 11, 1886.

Twenty Cases of Uncontrollable Vomitug, in which artificial abortion was Inducho. (Continued.)

\begin{tabular}{|c|c|c|c|c|c|c|c|c|c|}
\hline \multirow{2}{*}{$\dot{\leftrightarrow}$} & \multirow{2}{*}{ Reported by. } & \multirow{2}{*}{ Age. } & \multirow{2}{*}{ 遂是 } & \multicolumn{2}{|c|}{ Vomiting. } & \multirow{2}{*}{ Treatment. } & \multirow{2}{*}{$\begin{array}{l}\text { Termi- } \\
\text { nation. }\end{array}$} & \multirow{2}{*}{ Condition and Remarks. } & \multirow{2}{*}{ Reference. } \\
\hline & & & & Began. & Finded. & & & & \\
\hline 18 & $\begin{array}{l}\text { Dr. Engelmann, St. Louis, } \\
\text { Mo. }\end{array}$ & Young. & $\mathbf{P}$ & $?$ & 6th.mo. & Artiflcial abortion. & Death. & $\begin{array}{l}\text { " Patient died in eight to twelve } \\
\text { hours after abortion was pro- } \\
\text { duced. Vomiting was finally } \\
\text { stopped by application of car- } \\
\text { bolic acid to cervix and a tam- } \\
\text { pon of tannic acid, glycerine } \\
\text { and carbolic acid ; and it was } \\
\text { thought possibly the chances } \\
\text { might be better if the ovum } \\
\text { were removed." }\end{array}$ & $\begin{array}{l}\text { St. Louis Cour- } \\
\text { ier of Medi- } \\
\text { cine, 1884, 12, } \\
\text { p. 61-67. }\end{array}$ \\
\hline$\overline{19}$ & $\begin{array}{l}\text { S. G. Moses, M.D., St. } \\
\text { Louis, Mo. }\end{array}$ & $\begin{array}{l}\text { Under } \\
30 \\
\text { years. }\end{array}$ & $\overline{\mathbf{P}}$ & $?$ & $\begin{array}{l}\text { Between } \\
2 \mathrm{~d} \text { and } 3 \mathrm{~d} \\
\text { month. }\end{array}$ & $\begin{array}{l}\text { Antiflicial abortion. } \\
\text { Ovum punctured. }\end{array}$ & Death. & $\begin{array}{l}\text { Patient had been "vomiting for } \\
\text { flve or six weeks almost inces- } \\
\text { santly ; could retain absolute- } \\
\text { ly nothing." At time of abor- } \\
\text { tion patient was moribund, } \\
\text { feeble pulse, fetid breath and } \\
\text { foul tongue. Vomiting ceased } \\
\text { in twenty-five or thirty min- } \\
\text { utes. "Death from absolute } \\
\text { starvation." }\end{array}$ & $\begin{array}{l}\text { St. Louis Cour- } \\
\text { ier of Med., } \\
1884 \text {, 11, p. } \\
250-262 .\end{array}$ \\
\hline$\overline{20}$ & $\begin{array}{l}\text { S. G. Moses, M.D., St. } \\
\text { Louis, Mo. }\end{array}$ & $\begin{array}{l}\text { Under } \\
30 \\
\text { years. }\end{array}$ & $\overline{\mathbf{M}_{3}}$ & ? & $\begin{array}{l}\text { Between } \\
\text { 2d and 3d } \\
\text { month. }\end{array}$ & $\begin{array}{l}\text { Artificial abortion. } \\
\text { Ovum punctured. }\end{array}$ & Death. & $\begin{array}{l}\text { "Persistent vomiting." "Could } \\
\text { retain nothing." After abor- } \\
\text { tion, vomiting stopped. Patient } \\
\text { "actually starved to death." } \\
\text { Died in twelve hours. }\end{array}$ & $\begin{array}{l}\text { St. Louis Cour- } \\
\text { ier of Med., } \\
1884,11, \quad \text { p. } \\
250-262 .\end{array}$ \\
\hline
\end{tabular}

SUMMARY.

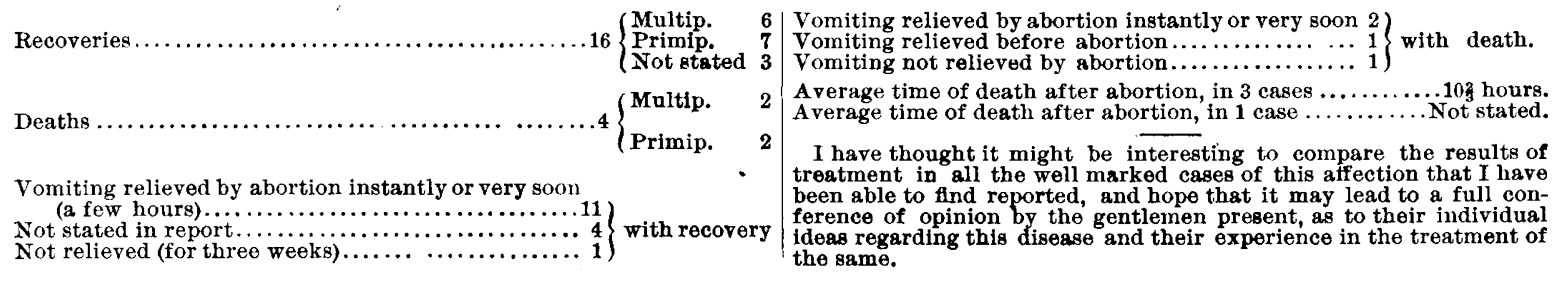

RECURRENT HEADACHE IN CHILDREN, AND ITS TREATMENT.

BY RUSSELL STURGIs, M.D.

IT is not uncommon to see children complaining of headache whose general health is apparently good. Such children usually are troubled with headache more or less severe, recurring at intervals of days or weeks, and each attack lasting from a few hours to a day or more. You will generally find that the child has been subject to these headaches for a year or more before treatment is sought for this particular symptom. As a rule, it will be noticed that the patients are of nervous temperament, that they do not sleep well, that they grind their teeth at night, and frequently suffer from bad dreams and nocturnal terrors. Frequently these children will have a spasmodic cough, particularly at night, even if there be no signs of pharyngitis or laryngeal irritation to account sufficiently for such cough. It may be noticed that the child has difficulty in keeping its hands quiet, that if told to keepstanding quietly it will constantly change its weight from one foot to the other. In temper the child is apt to be fretful. Nothing particular is to be noticed about the face with the exception of a peculiar heavy expression about the eyes. This expression is noticed in people with migraine, and also in any depressing illness. Dr. Warner, ${ }^{1}$ after careful examination, thinks this expression is due to lack of tone in the orbicularis palpebrarum, giving "an appearance of flabbiness about the lower eyelid; the skin hangs too loose with an increase in the number of folds, and in place of falling neatly

1 Physical expression. against the lower eyelid as a convex surface, it falls more or less in a plane from the ciliary margin to the lower margin of the orbit, a condition often best seen in profile." This heavy expression about the eyes is not noticed in all cases of recurrent headaches.

These headaches generally come on in the morning, though the child may be perfectly well on going to bed the night before. The pain is generally localized in some particular part of the head, either side, frontal region or vertex. The pain is severe, during the seizure, usually preventing study or play. It is not unusual for there to be optical illusions of sparks, or bands and spots of color. There may or may not be nausea during the attack. The child generally feels cold, and in winter prefers to lie near the stove huddled up in a chair.

The picture is very like that of migraine in the adult, with this difference : the pain is more apt to be across the forehead and may be at the base or at the vertex of the skull; there is rarely vomiting or nausea with the attack, and such attacks seem to yield to a treatment which is of very little use in hemicrania of the adult.

Dr. Eustace Smith in his latest book, considers the headaches identical with migraine. Dr. Warner is inclined to think that these recurrent attacks are but a mild form of chorea. It has been shown by Dr. Hughlings Jackson and by Dr. Herman that in cases of chorea about seventy-five per cent. of the children suffered from recurrent headaches.

Since 1882 I have seen sixteen cases of recurrent headache. At first I spent a long time unsuccessfully in trying to relieve the patient by attention to diet and 